\title{
DERECHOS DE LOS MÁS DÉBILES. EL LIBERALISMO IGUALITARIO FRENTE A LOS PROBLEMAS DE LA MINORIDAD
}

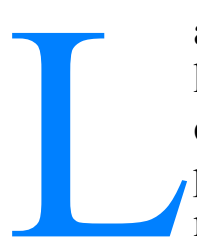

a intuición básica que motiva este trabajo es la siguiente: resulta difícil de aceptar el hecho de que una persona menor de edad, no siendo en absoluto responsable de las capacidades con las que nace dotada, o del contexto dentro del cual va a crecer, puede verse condenada toda su vida (o buena parte de ella), a tratar de revertir o moderar las penurias derivadas de aquellas condiciones iniciales ajenas a su control.

Para analizar el tipo de problemas vinculados con cuestiones como la arriba planteada, seguiré en este trabajo dos estrategias. Por un lado, analizaré las respuestas que la justicia constitucional ha dado, en diferentes materias que conciernen a los derechos de los/las menores. En particular, voy a estudiar diferentes soluciones propuestas por la Corte Suprema de los Estados Unidos. Las razones para adoptar esta primera estrategia son varias: i) Los argumentos presentados en las sentencias de aquel tribunal suelen recoger y elaborar, muy sofisticadamente, las mejores teorías legales en curso; ii) tales sentencias ilustran, de un modo notable, de qué modo, una democracia estable y desarrollada, tiende a resolver problemas como el de la minoridad, aquí planteado; iii) los pronunciamientos de tal tribunal resultan habitualmente de enorme influencia, al menos, en todo el mundo occidental.

Esta primera estrategia, sin embargo, es claramente limitada. El conocer de qué modo un tribunal (normalmente) tan lúcido como el norteamericano suele fallar sus casos, sólo nos da algunas pistas acerca de soluciones razonables y posibles para enfrentar problemas como los aquí implicados. No hace falta que enfatice, de todos modos, que tales soluciones pueden resultarnos perfectamente inaceptables. De hecho, una de las intenciones de este trabajo será la de mostrar los límites y falencias que, frecuentemente, aparecen en los fallos de aquel tribunal. Quiero decir, me interesará mostrar que los problemas de la minoridad, muchas veces, son resueltos de modo objetable. Para efectuar este análisis crítico, entonces, es que recurriré a la anunciada segunda estrategia. Para ello, partiré de una cierta postura ideológica, el liberalismo igualitario, y desde allí procuraré evaluar posiciones 
como las presentadas por la Corte Suprema norteamericana. En este sentido, asumiré como correctas muchas de las intuiciones básicas corrientemente defendidas por el liberalismo igualitario.

A los fines de mi exposición, en primer lugar, aclararé qué es lo que voy a entender por liberalismo igualitario. Voy a definir tal postura a partir de ciertas nociones convencionales al respecto, y contraponiéndolo con otras posiciones menos liberales o menos igualitarias. Establecidas estas distinciones iniciales, voy a comenzar el prometido análisis crítico de la jurisprudencia norteamericana, respecto de la situación de los menores. En mi estudio, según lo dicho, trataré de utilizar los criterios que podría adoptar un liberal igualitario. De todos modos, y dadas las naturales vaguedades e imprecisiones que rodean a temas como los que voy a abordar, asumir tal punto de vista ideológico no me permitirá dar respuesta clara (¡como si la hubiera!) a todos los interrogantes que trataré. Lejos de ello, me veré obligado, muchas veces (y debido a las mencionadas incertezas), a elaborar cuáles podrían ser las «respuestas liberales igualitarias ante los distintos problemas a examinar»; o, aún, a re-elaborar las posiciones aparentemente adoptadas por los liberales igualitarios, de modo de hacerlas más coherentes con sus presupuestos centrales.

\section{Liberales igualitarios y no igualitarios}

Para delimitar, aproximadamente, qué es lo que voy a entender cuando hable de liberalismo igualitario, voy a apoyarme en ciertos criterios más o menos convencionales al respecto. Estos criterios, me permitirán distinguir, al menos a grandes rasgos, entre una postura más propia del liberalismo igualitario, de otras más cercanas al liberalismo clásico o conservador ${ }^{1}$.

i) Una primera forma de distinguir entre posturas igualitarias y conservadoras, se vincula con el modo en que unas y otras evalúan la distribución de los recursos iniciales en una sociedad. Para el liberalismo conservador, un cierto sistema institucional es considerado justo en tanto y en cuanto la distribución de recursos que resulte del mismo sea el producto de las decisiones de un mercado económico eficiente, en el cual prevalezca un principio de igualdad de oportunidades talque todas las posiciones se encuentren abiertas a aquellos dotados con los talentos relevantes ${ }^{2}$. El liberalismo igualitario, en cambio, sostiene que debe actuarse «correctivamente» sobre la

${ }^{1}$ En lo que sigue, denominaré a las posturas no igualitaristas, indistintamente, posturas «conservadoras», «liberales clásicas», o «liberales no igualitarias».

${ }^{2}$ Ver, principalmente, Robert Nozick, Anarchy State and Utopia (Oxford, 1974). Comparando posturas igualitarias y no igualitarias ver, especialmente, Michael Sandel, Liberalism and the Limits of Justice (Cambridge U.P., 1982); o Philippe Van Parijs, Que es una sociedad justa (Barcelona, 1993). 
inicial distribución de recursos existente en la sociedad. Ello, por considerar que aquella distribución originaria, estuvo decididamente influida por contingencias sociales y naturales fuera del control de los individuos (producto de la «lotería natural») ${ }^{3}$, y que tales individuos no deben ser beneficiados o perjudicados por hechos de los que no son responsables. Muchos conservadores llegan a admitir, junto a los liberales igualitarios, la arbitrariedad moral que caracteriza a aquella distribución inicial. Sin embargo, se niegan normalmente a autorizar actuaciones del Estado sobre tales arbitrariedades ${ }^{4}$.

ii) El liberalismo igualitario se caracterizaría, además, por entender que los derechos de las personas pueden ser violados tanto por acciones de otros como a partir de comportamientos omisivos (con lo que, paralelamente, el liberalismo igualitario muestra su preocupación tanto por asegurar la llamada «libertad negativa» de las personas, como también por promover lo que se entiende su «libertad positiva»). En este sentido, reconocería la existencia de ciertos derechos «positivos» (o sea, derechos que tienen como correlato obligaciones activas por parte de los demás, como podría serlo el derecho a una alimentación adecuada $)^{5}$. El liberalismo conservador, en cambio, aparecería más bien renuente a reconocer la posibilidad de que se violen los derechos de alguna persona a través de conductas omisivas ${ }^{6}$. Más bien, estaría concentrado en defender un principio de no agresión por parte de terceros (y en particular del Estado), respecto de los derechos «negativos» de las personas (o sea, derechos que tienen como correlato obligaciones de conductas pasivas por parte de los demás).

iii) Contemporáneamente, para distinguir entre los dos tipos de liberalismo mencionados, se suele recurrir a un criterio adicional. Este criterio se vincula con el distinto modo en que igualitarios y conservadores se aproximan a una idea que ambos utilizan como punto de partida de sus doctrinas: la idea de libre elección, o elección autónoma. Los liberales no igualitarios, en este sentido, se caracterizarían por asumir como «dadas» las preferencias de los distintos individuos; mientras que los igualitaristas se distinguirían en su preocupación por la «formación endógena» de esas preferencias. Con esta última idea quiero decir que los liberales igualitarios no están dispuestos a calificar como autónomas ciertas preferencias que han sido «moldeadas» a partir, por ejemplo, de la falta de información relevante;

${ }^{3}$ Ver, principalmente, John Rawls, A Theory of Justice (Cambridge. Mass., 1971)

${ }^{4}$ En este respecto, y comparando el igualitarismo de Rawls con el conservadurismo de Robert Nozick, ver Philippe Van Parijs, Que es una sociedad justa. Barcelona, 1993, p. 162.

5 Ver, por ejemplo, Carlos Nino, The Ethics of Human Rights Oxford U.P., 1991); Jonathan Glover, Causing Death and Saving Lives (Harmondsworth, 1977); Onora O Neill, How Do We Know When Opportunities Are Equal?, incluido en Feminism and Philosophy, ed. por Mary Vettecling-Braggin; Frederick A. Elliston; Jane English (Rowman and Littlefield, N. Jersey, 1977).

${ }^{6}$ Ver, en particular, Carlos Nino The Ethics. 
de defectos tales como la debilidad de voluntad; la «miopía» tendencias adaptativas; etc. ${ }^{7} \mathrm{Al}$ nivel de las elecciones sociales (que son las que aquí más nos interesan), los liberales igualitarios tienden a asociar la idea de elecciones autónomas con aquellas que surgen luego de un proceso colectivo de discusión «racional y pública» ${ }^{8}$. Este proceso de discusión es valorado, entre otras cosas, en tanto puede contribuir a «transformar» preferencias meramente basadas en prejuicios; errores fácticos; interpretaciones equivocadas de las preferencias de los demás; etc. Las ideas aquí expresadas, de modo más que sintético, han sido desarrolladas larga y profusamente en los últimos años, por quienes -diría- defienden una concepción «deliberativa» de la democracia".

Los liberales no igualitarios, en cambio, parecen defender una visión diferente de la democracia (llamada a veces «pluralista» $\mathrm{o}$ «economicista»), que parte del tomar las preferencias de los individuos como «privadas y exógenas» ${ }^{10}$. Esto es, los liberales más clásicos se desentienden del proceso por el cual las preferencias en cuestión fueron moldeadas. Ello puede deberse, tanto a su temor ante las consecuencias negativas posibles del «revisar» el origen de las preferencias; como a su confianza en la libertad del individuo por cambiar sus deseos, ante el conocimiento de posibilidades diferentes. Para considerar un sistema de selección de preferencias como legítimo, en este caso, bastaría con que tales preferencias («dadas») se hayan podido «agregar» colectivamente, a través de mecanismos tales como el sufragio mayoritario ${ }^{11}$. Diferencias como las hasta aquí señaladas permitirían, por ejemplo, asociar a los liberales igualitarios con la defensa persistente de

7 El decir que estas elecciones no pueden considerarse, genuinamente, como autónomas, no implica suscribir, necesariamente, a una posición de tipo kantiana, en esta materia. Ver este análisis sobre la formación endógena de las preferencias, y un novedoso acercamiento a la idea de autonomía, fundamentalmente, en Jon Elster, Belief Bias and Ideology, incluido en el libro Rationality and Relativism, ed. por Martin Hollis y Steven Lakes (Blackwell. Cambridge, 1993); Sour Grapes (Cambridge U. P., 1g83); o The Market and the Forum, incluido en Foundations of Social Choice Theory, ed. por J. Elster y A. Hylland (Cambridge, 1989), p. 107.

${ }^{8}$ Ver, por ejemplo, Jon Elster, op. cit. págs. 111-112.

${ }^{9}$ En este sentido ver, por ejemplo, Joshua Cohen, Deliberation and Democratic Legitimacy, en the Good Polity, ed. por A. Hamlin y P. Petit; Carl Sunstein, The Partial Constitution (Harvard U. P. 1993); Carlos Nino, Etica...; Bernard Manin, «On Legitimacy and Political Deliberation» Political Theory, vol. 15, $\mathrm{n}^{\circ} 3$ (agosto, 1987): 338-368; Amy Gutman y D. Thomson, «Moral Confliat and Political Consensus» Ethics 101 (octubre, 1990): 64-68: etc.

10 Cass Sunstein, «Naked Preferences and the Constitution, «Columbia Law Review, vol. 84, n. 7 (Noviembre de 1984), criticando esta postura. En el mismo sentido, ver, Cass Sunstein, «Beyond the Republican Revival». 97 Yale Law Journal, 1539.

11 James Madison podría ser incluido como un primer relevante defensor de esta postura «pluralista» de la democracia. Otros han hecho referencia también a autores más contemporáneos, como Joseph Schumpeter, Democracy Capitalism and Socialism (N. York, 1976); o al Robert Dahl de A Preface to Democratic Theory (Phoenix Books, the University of Chicago Press, 1963). 
foros de discusión pública; a la vez que asociar a los no igualitaristas con la defensa central de la institución del mercado.

Las diferencias anteriores, según intentaré mostrar, resultan cruciales para advertir el diferente modo en que unos y otros liberales enfocan el tema de la niñez. Para los liberales igualitarios, resulta necesario «liberar» a los menores de todas aquellas cargas iniciales de las que no son en absoluto responsables (incapacidades físicas; falta de medios económicos) y educarlos en y para la discusión pública. Para los liberales más clásicos en cambio, el énfasis debe estar puesto en educar a los menores para que puedan desempeñarse en el mercado. A continuación, voy a tomar en cuenta distintos casos resueltos por la Corte Suprema norteamericana, vinculados a la problemática de la minoridad, y voy a examinar tales fallos críticamente, mostrando de qué modo se reproduce en ellos la tensión entre un pensamiento igualitarista, y otro más bien conservador.

\section{Discriminación: Separados pero iguales}

El primero de los temas que voy a estudiar tiene que ver con el acceso a la educación, y las discriminaciones que aparecieron en dicho respecto.

El liberalismo, por distintas razones, siempre se preocupó por asegurar que todos los individuos tuviesen acceso a la educación. Sin embargo, tal preocupación no siempre se extendió a asegurar condiciones mínimamente aceptables para esa enseñanza. Un ejemplo notable al respecto, puede localizarse en los E.E.U.U., al menos hasta bien entrado este siglo, donde se entendía que blancos y negros tenían ambos derecho a estudiar pero no necesariamente, por ejemplo, a estudiar en los mismos establecimientos educativos. Para ser más específico, se consideraba moralmente aceptable que existiesen escuelas de blancos, en las que se prohibía la entrada de niños negros, en tanto y en cuanto estos últimos pudiesen encontrar otras escuelas en donde estudiar.

La justicia norteamericana, que se ha caracterizado por albergar a muchas de las mentes más lúcidas de dicha sociedad, toleraba cómodamente la situación arriba descripta. El principio con el que resumían su postura al respecto, era el de «separados pero iguales». Con esto querían significar, en pocas palabras, «la discriminación puede ser aceptable, en tanto los negros puedan acceder a facilidades similares en otro lugar». La idea de «separados pero iguales», apareció explicitada por primera vez en el caso «Plessy v. Ferguson», (163 U.S. 537, en 1896), y en él se aceptó la constitucionalidad de un estatuto de Lousiana, que adoptaba dicho principio, en materia de transporte público. En el área educativa, la aplicación de una idéntica provisión importó soluciones como la siguiente: tolerar prácticas discriminatorias (como la de que los blancos no le permitan a los negros compartir ámbitos de discusión comunes), a la vez que se obligaba a ciertos estados, por 
ejemplo, a proveer de educación a las personas de color discriminadas. De este modo, los jueces aparecían jugando un papel muy activista (al prescribir, como en algunos casos, la construcción de escuelas para la gente de raza negra $)^{12}$ mientras aceptaban como moralmente admisibles prácticas de rechazo racial.

Fueron ideales más igualitarios, entiendo yo, los que comenzaron a cuestionar los serios límites a que se veían sometidos los negros en cuanto a sus posibilidades educativas. En especial, tales ideales comenzaron a poner contra las cuerdas principios tales como el de «separados pero iguales»».

La visión igualitarista, según la presenté más arriba, centra buena parte de sus preocupaciones institucionales en la idea de deliberación colectiva; a diferencia de la visión más clásica, preocupada más específicamente en capacitar a los individuos para su entrada al mercado. De modo coherente con este ideal de diálogo, muchos jueces (que yo describiría entonces como «más igualitarios»), objetaron, desde fines de la década del 40, la separación racial hasta entonces autorizada. Así fue como, por ejemplo, se fundamentó la resolución del caso «Sweatt v Painter» (339 I.S. 629, de 1950). En dicho caso, y de manera coincidente con el ideario deliberativo del liberalismo igualitario, la Corte sostuvo que ningún alumno elegiría estudiar en un ámbito académico vaciado, «distanciado del interjuego de ideas, y del intercambio de puntos de vista». La Corte reafirmó tales principios, prontamente, en el caso «McLaucin v. Oklahoma State Regents» (339 U.S. 637, de 1950). Al estudiante negro McLaucin, se le impedía no sólo compartir una clase, sino también tomar café, o estudiar en la biblioteca, junto con los blancos. La Corte, frente a dicha situación, sostuvo la inconstitucionalidad de tales medidas porque empeoraban e inhibían la posibilidad de que McLaucin estudiase, se «trabase en discusiones e intercambiara opiniones con otros estudiantes».

El punto más alto de esta tradición igualitaria (vinculada con la no discriminación en los ámbitos educativos), estuvo dada por el famoso fallo «Brown v. Board of Education of Topeka» (347 U.S. 483, de 1954), llevado adelante por el juez Th. Marshall (que luego se convertiría en juez de la Corte Suprema, y llegaría a ser uno de los miembros más igualitaristas dentro de la historia del tribunal). Conforme al pensamiento liberal más clásico,

12 'En «Gaines v. Canada,» (305 U.S. 337, de 1938), por ejemplo, la Suprema Corte obligó al estado de Missouri a asegurar el derecho a una «igual protección de las leyes» para los negros, proveyéndolos de facilidades educacionales «substancialmente iguales» a aquellas que se proveían a los blancos (aún esta débil idea de igualdad era relativizada, sin embargo, al permitírsele a los agentes estatales mismos el determinar si las facilidades creadas eran «lo suficientemente iguales,» siempre y cuando tales agentes actuasen con «buena fe»y razonabilidad. Esta idea quedó en claro, al menos, desde el caso «Cumming v. Board of Education,»175 U.S. 528, de 1899.) Esta igualdad, obviamente, era igualdad en la no integración. Similarmente, en «Sipuel v. Board of Regents» (332 U.S. 631, de 1948), la justicia ordenó ya sea la aceptación de Sipuel (un estudiante negro), en el mismo ámbito que sus pares blancos, ya sea el pronto establecimiento de una institución idéntica para los de raza negra. 
la Corte sostuvo que, en efecto, la educación constituía «tal vez la función más importante del gobierno de la nación y de los estados». Sin embargo, y ya más a tono con nuevos ideales igualitarios, citaba el fallo McLaurin para resaltar la necesidad de la no segregación de las personas de distinta raza en establecimientos separados, a fin de permitir el debate, y la confrontación de opiniones entre los diversos estudiantes. La idea de «separados pero iguales» -concluía el fallo- ya no podía tener lugar. La idea de «facilidades educacionales separadas» -se sostuvo- era inherentemente desigualitaria. Este fallo «Brown», no hace falta decirlo, pasó a constituirse en el símbolo de la nueva «revolución judicial» norteamericana. De algún modo, también, se constituyó en uno de los puntos de ruptura más significativos entre dos tradiciones liberales seriamente opuestas. Hoy, seguramente, ninguna persona razonable se animaría a defender los argumentos que, en materia de discriminación de estudiantes de raza negra, eran usuales en la época previa al caso «Brown». Sin embargo, intentaré mostrar a continuación que muchos de los presupuestos de aquellas doctrinas defectuosamente liberales o igualitarias siguen vigentes en otras áreas.

\section{El derecho a «escuchar otras músicas»}

Otra grave cuestión vinculada a los derechos de los menores de edad, tiene que ver con los contenidos de la educación (formal e informal) que van a recibir, especialmente en sus primeros años. Aparentemente, tal formación resulta determinante para la vida futura de los niños y de allí, obviamente, la preocupación por ver si alguien monopoliza la palabra y la enseñanza en aquellos años.

Respecto de los mencionados contenidos, los liberales igualitarios han marcado su posición al poner un especial énfasis (más que en alguna especial visión substantiva sobre lo que los niños debieran creer, o sobre los fines que debieran perseguir), en lo que podría ser llamada una concepción del bien de segundo orden ${ }^{13}$. Esto es decir, por ejemplo, que se debe formar a los menores de un modo no dogmático, abierto al reconocimiento de diferentes valores o ideales del bien. El énfasis igualitario no está puesto en el aprendizaje de algún punto de vista en particular, pero sí en la necesidad de que se conozcan distintos puntos de vista. Las intuiciones que se mueven detrás de esta concepción igualitarista, pueden tener que ver con las siguientes. Por un lado, con el hecho de darle la oportunidad a los menores de elegir del modo más amplio posible cuál es el modelo de vida futura que quieren perseguir. En este sentido, se remarcaría la idea de que los padres no deben precluir a sus hijos e hijas la posibilidad de conocer planes de vida

13 En sentido similar, ver, por ejemplo, Brian Barry, «Derechos Humanos, individualismo y escepticismo» Doxa, 1992, p. 224. 
alternativos. Quiero decir, metafóricamente, que los padres no deben privar a su descendencia del derecho de «escuchar otras músicas». En un mismo sentido, el igualitarismo ha señalado siempre, frente a sus críticos, que los menores no deben ser vistos como «propiedad» de sus padres ${ }^{14}$ y que, por el contrario, y en la medida en que maduran, deben tener un derecho creciente a ser escuchados (aún a riesgo de contradecir la voluntad de los padres), en todo lo que haga a la construcción de su propio futuro. Adicionalmente, los liberales igualitarios asumen la necesidad de que se prepare a los/las menores para su propia participación en el diálogo democrático futuro, al que van a incorporarse ${ }^{15}$. Este tipo de intuiciones, creo yo, no son incompatibles con la posibilidad de que los padres instruyan a sus hijos e hijas en determinadas creencias o concepciones del bien que ellos juzgan más valiosas. En cambio lo que sí se rechaza es que dichos padres impidan a los menores acceder a valores alternativos.

Para llevar a cabo convicciones como las citadas, el liberalismo igualitario ha tendido a defender un papel activo del Estado, destinado a maximizar la autonomía de los individuos, en aquellos casos en que ésta pueda ser cercenada, o puesta en peligro.

El liberalismo más clásico, en cambio, y según vimos, parece menos interesado en revisar el modo en que tales preferencias se han formado hasta, de alguna manera, desentenderse casi por completo de dicha cuestión. Al liberal le resulta, en principio, suficiente con conocer que para determinado individuo el modelo de autorrealización x es preferible al z, más allá de cuáles hayan sido las razones de tal elección, y más allá del «grado de autonomía» con que tal elección se haya llevado a cabo.

Posiblemente, lo que está detrás de esta postura es una convicción acerca de la fuerza persuasiva de las ideas. Se podría pensar, en este sentido, que según el liberalismo clásico, más allá de la formación dogmática u opresiva que alguien haya podido recibir, el solo contacto con la luz de mejores ideas basta para borrar toda una historia de oscurantismo ${ }^{16}$. De allí que no

${ }^{14}$ Por ejemplo, y especialmente, en los casos enmarcados dentro de la idea de «child labor» (o explotación económica de los menores), rotundamente repudiados por la justicia. Ver, en este sentido, «Hammer v Dagenhart» (247 U.S. 251, de 1918). Sin embargo, y en forma coherente con la idea de que «el menor pertenece a los padres», la Corte llegó a tolerar inaceptables maltratos de los padres sobre los hijos. Al respecto, ver Akhil Amar y Daniel Widawsky, «Child Abuse as Slavery». Harvard Law Review, vol. 105, n. 6 (1992), en el cual los autores comentan el caso «De Shaney v. Winnebago County Department of Social Service» (489 U.S. 189, de 1989), en el cual la Corte sostuvo que el estado de Wisconsin no tenía el derecho constitucional de proteger a un menor frente el abuso físico de su padre.

15 Bruce Ackerman, Social Justice in the Liberal State. Yale U. P, 1980, p. 140. En el mismo texto, Ackerman señala algunos de los límites de su posición, que no lo llevan, por ejemplo, a predicar la necesidad de educar a los niños en «una infinita y cambiante Babel de discursos», capaz de saturar la misma capacidad de comprensión de los menores. Op. cit. p. 141.

${ }^{16}$ De hecho, este tipo de posiciones son las que, de alguna manera, han procurado defender liberales como Richard Epstein, frente a teóricos más igualitarios, como Jon Elster o Cass Sunstein, que mostraban su preocupación por lo que llamamos proceso endógeno de formación de las preferencias. Según Epstein, sucesos como los acontecidos en los ex-países socialistas de Europa del Este, ejemplifican claramente la plausibilidad de su postura. En aquellos países, años de formación totalitarista, por ejemplo, no obstaron a que los ideales del libre mercado (como los que él defiende) se hayan inmediatamente impuesto, apenas se resquebrajaron las paredes del adoctrinamiento comunista. Richard Epstein, «Two Conceptions of Civil Rights», Social Policy and Philosophy, vol. 8, n. 2. 
haya ninguna preferencia que «corregir», ninguna decisión que «mejorar». El riesgo que existiría, si es que alguno, sería el de que el Estado se convierta en omnipresente, pretendiendo, por ejemplo, monopolizar el control de la educación. En definitiva, las posiciones más conservadoras consideraron al activismo estatal en materia educativa como indeseable (dado que nada había para «corregir»); y además como inconveniente (dados los riesgos propios implicados en la creación de cualquier aparato de control o supervisión).

En la jurisprudencia norteamericana, posiciones como las descriptas quedaron bastante fielmente reflejadas. Por un lado, liberales igualitarios y conservadores coincidieron, en un común ánimo por asegurar la libertad de cátedra ${ }^{17}$. Sin embargo, inmediatamente, tales acuerdos se convirtieron en disidencias en cuanto a qué papel permitirle (o exigirle) jugar al Estado, frente a los derechos de los menores, y las preferencias de los padres. La línea que predominó, respecto de este punto, fue la caracterizada por una fuerte hostilidad hacia el Estado. Tal actitud pudo observarse, en primer lugar, a través de una cierta asociación entre la idea de pluralismo, y la existencia de escuelas privadas. Esta asociación (incluso defendida por juristas partidarios de la intervención estatal) ${ }^{18}$ tendió a ver en la mera existencia de escuelas públicas un alto riesgo de indoctrinamiento, que se entendió básicamente salvado a partir de la existencia de escuelas privadas. Quiero decir, se consideró que el pluralismo estaba esencialmente satisfecho con la coexistencia de escuelas públicas y privadas, dejando más bien a un lado la cuestión de los contenidos específicos de la enseñanza impartida en tales ámbitos. En los famosos casos «Meyer»y «Pierce» ${ }^{19}$ la Corte puso de manifiesto sus lineamientos esenciales en la materia, afirmando una primacía en la «libertad de los padres y tutores para dirigir el crecimiento y la educación de los menores bajo su control ${ }^{20}$.

${ }^{17}$ En este sentido, la Corte se pronunció infinidad de veces. Así, por ejemplo, al revertir la sanción a un educador que había distribuido un material didáctico que contenía algún vocablo aparentemente obsceno ( «Keefe v. Geanakos», 418 F.2d 359, de 1969); ordenando la reincorporación de un profesor que había asignado la lectura de un cuento de Vonnegut, que se consideraba inadecuado («Parducci v. Rutland», 316 F. Supp. 352, 355, de 1970), aceptando que una junta educativa separase 10 libros, sobre una lista que superaba el millar, con lo cual reafirmaba el derecho de los maestros a una completa «libertad de expresión en las aulas» (Cary v. Board of Education, 593 F.2d 535, 543, de 1979); etc. 1988), p. 812 .

${ }^{18}$ Ver, por ejemplo, Laurence Tribe, American Constitutional Law, (New York, the Foundation Press, Inc.,

19 «Meyer v. Nebraska» (262 U.S. 390, de 1923); y «Pierce v. Society of Sisters» (268 U.S. 510 de 1925).

20 Este sesgo antiestatal se reafirmó, por ejemplo, en el caso «Tinker v. Des Moines Independent Community School District» (393 U.S. 503, 511, de 1969). 
La Corte también se opuso a que el Estado promoviese, dentro de las escuelas, un tratamiento «equilibrado» de puntos de vista opuestos, tratamiento equilibrado que implicaría que, frente a determinados tópicos, los distintos puntos de vista existentes sean expuestos. Este criterio, por ejemplo, fue discutido respecto de un estatuto de Louisiana que obligaba a presentar dicha pluralidad de opiniones en las escuelas, cuando en ellas se trataba el tema del origen de la vida (en este caso, tal principio implicaba presentar tanto la doctrina de la «creación», como la doctrina «evolucionista») ${ }^{21}$. Más allá de las razones que la mayoría pudo tener en este específico caso, para fallar de este modo, también pudo percibirse aquí una hostilidad más de fondo hacia el principio «del equilibrio,» que el pensamiento igualitarista podría aprobar con cierta satisfacción.

\section{Formación pluralista y comunidades de valores uniformados}

Pero el caso seguramente más difícil, de entre toda esta línea jurisprudencial adversa al Estado, pudo verse en «Wisconsin v. Yoder» (406 U.S. 205, de 1972). En esta ocasión, estaba en juego la voluntad de los padres de un menor de 14 años, pertenecientes a la comunidad religiosa Amish. Dichos padres, (conforme al pensamiento de los miembros de la comunidad), entendían que, luego de haber recibido una cierta educación elemental, ya no era necesario mantener al joven dentro del sistema educativo. Por el contrario, de acuerdo con la opinión de los padres, el joven ya gozaba de una instrucción suficiente, que le permitía cumplir satisfactoriamente con las futuras tareas que desempeñaría dentro de la orden religiosa. Por ejemplo, ya sabía escribir, y ya era capaz de leer la Biblia. Lo demás, entonces, era inútil, y más bien ligado al indoctrinamiento en valores que no eran los compartidos por los Amish. Quedaba en juego, entonces, y en primer lugar, una ley del estado de Wisconsin, que determinaba la obligatoriedad de la enseñanza hasta los 16 años.

La situación, en verdad, no configuraba un caso especialmente «difícil». En última instancia, sólo estaban en juego dos años de enseñanza obligatoria y ello, entre otras razones (por ejemplo, el hecho de que los Amish constituyeran un grupo largamente establecido, y dedicado a fomentar ideales cooperativos en el trabajo rural), movió a la Corte a apoyar los reclamos efectuados por los padres del menor. De todos modos, resulta claro, bullían detrás de tal decisión cuestiones mucho más dramáticas, que fueron las que llevaron a muchos liberales igualitarios a escandalizarse con los principios enunciados en la resolución del caso. Dos temas, en particular, irritaron a los pensadores igualitarios. 1) Por un lado, el rol determinante que se asignaba a la voluntad de los padres (de un modo coherente con la línea que se

21 «Edwards v. Aguillard» (107 S.Ct. 2573, de 1987). 
venía desarrollando en casos como los ya vistos); y 2) la prioridad que se le asignaba a un valor como el de la autonomía religiosa, frente a otros bienes más básicos, como el de estar en condiciones de elegir qué orientación religiosa adoptar, si es que alguna.

Respecto de la primera cuestión, el pensamiento igualitarista no sólo invocó los derechos del Estado respecto de la regulación de las cuestiones educativas; sino además, y fundamentalmente, los derechos del menor frente a sus padres. Este criterio ya había tomado cuerpo en la opinión disidente del juez Douglas, en el caso «Yoder», cuando señaló que era «el futuro del estudiante, y no el futuro de los padres», el que resultaba puesto en peligro por la decisión que se acababa de tomar. Es el niño -sostuvo- el que a través de este tipo de decisiones va a ver puesto en serio riesgo «su capacidad para entrar en contacto con un nuevo y sorprendente mundo pleno en diversidad. El niño puede entender que el curso preferible es el determinado por los padres, o puede rebelarse frente a dicho curso. Es el juicio del estudiante, y no el de los padres, el que está en juego». En modo similar, el teórico igualitario Bruce Ackerman señaló que el Estado debía dar creciente importancia a los juicios de los menores a medida en que crecen, y muy en especial respecto de cuestiones tales como qué mensajes ellos consideran preferible atender ${ }^{22}$. Amy Gutman, también, señaló acerca del mismo caso que, cuando «un menor es suficientemente maduro para distinguir sus creencias religiosas de aquellas de sus padres, sus creencias deben ser consultadas ${ }^{23} \gg$.

Ahora bien, y respecto de la segunda de las cuestiones citadas, el igualitarismo siempre prestó particular atención al modo en que los individuos deciden acerca de sus planes de vida. Para un liberal igualitario, conforme hemos venido advirtiendo, existen ciertas precondiciones elementales para el desarrollo de una vida autónoma, que toda comunidad debiera asegurar a sus habitantes. En especial, toda sociedad debiera preparar a sus miembros no sólo para ser capaces de elegir entre diferentes concepciones del bien, sino además, y fundamentalmente, para poder discutir con los demás miembros de la comunidad de qué modo van a orientar sus vidas en el futuro $^{24}$. Si, en este sentido, la comunidad no asegura que el menor en cuestión pueda reconocer otros valores alternativos, frente a los que los padres le inculcan, esa comunidad cometería una omisión reprochable. Se quiere decir, si

${ }^{22}$ Bruce Ackerman, op. cit. cap. 5.

23 Amy Gutman, «Children, Paternalism, and Education: A Liberal Argument». Philosophy and Public Affairs 9, n. 4, 1980, p. 355. De todos modos, entiendo yo, el cuerpo teórico igualitario debe trascender aún este primer peso, para ser coherente con su preocupación acerca del modo en que las mismas preferencias del menor fueron moldeadas. Esto es, para un liberal igualitario, por ejemplo, la opinión de un esclavo que ha sido educado (él, y todos sus antepasados), en esa condición, tiende a perder peso relativo, por más que el esclavo en cuestión insista en su preferencia por continuar siendo esclavo.

${ }^{24}$ De modo similar, destacando estos dos principios, ver Amy Gutman, op. cit., p. 349. 
ese menor no ha estado en contacto con una diversidad de ideas religiosas o morales, entonces, difícilmente podría decirse que tal individuo está haciendo uso de su libertad de conciencia cuando adscribe a una determinada postura. Tal libertad, entiende el igualitarismo, sólo puede ejercerse en la medida en que tal persona haya sido educada en el conocimiento de pautas diferentes a las que sus padres le inculcan ${ }^{25}$.

El filósofo igualitario Joseph Raz parece apuntar a consideraciones similares a las aquí expuestas, en un minucioso estudio sobre la idea de autonomía. Según Raz, una decisión no puede ser considerada autónoma si quien la toma no la elige de entre «un adecuado abanico de opciones» ${ }^{26}$. El menor, en este caso, perdería irremediablemente parte de su capacidad (presente $\mathrm{y}$, sobre todo, futuro) de decidir libremente, si sus padres no lo educaron en el conocimiento de una multiplicidad de opciones posibles ${ }^{27}$.

En definitiva, para el igualitarismo, una sociedad justa debería proveer una educación pluralista. Sin embargo, para esta postura, el pluralismo no se garantiza, meramente, asegurando la existencia de escuelas públicas y privadas ni, mucho menos, reduciendo el papel que el Estado puede jugar. Para analizar si una cierta educación es pluralista se requiere examinar los particulares contenidos que se imparten, en cualquier esfera: el hogar, la escuela pública o la escuela privada. El Estado, luego, deberá tomar cartas en el asunto cada vez que una cierta comunidad o grupo impida a sus miembros, directa o indirectamente, el conocer otros valores más allá de los que en tales ámbitos se promueven.

\section{Educación de los pobres y educación pobre}

El tema que más particularmente me interesa tratar, respecto de la cuestión de la minoridad, tiene que ver con el impacto de las variables económicas

$25 \mathrm{Al}$ defender este punto de vista, Amy Gutman entiende estar desafiando el primer principio rawlsiano de prioridad absoluta de la libertad. En este caso, -sostiene Gutman- la libertad religiosa debe restringirse de modo de poder crear las condiciones para que esa misma libertad religiosa sea posible. Ver A. Gutman, Children...

26 Joseph Raz, The Morality of Freedom. Oxford U.P., 1989, p. 373. Raz hace referencia también a otras dos condiciones adicionales, para considerar a una decisión como autónoma. Una, la de que quien decide tenga una habilidad mental suficiente como para formar intenciones de una cierta complejidad, y planear su ejecución; y otra, la de que la decisión haya estado libre de coerción y manipulación, o sea, que haya sido una decisión «independiente».

27 De hecho, en trabajos más recientes Raz ha sido aún más explícito al respecto, señalando que en una sociedad multicultural, los niños «deben ser educados, si sus padres así lo desean, en la cultura propia de su grupo. Sin embargo, todos ellos deben también ser educados de modo tal que conozcan la historia y las tradiciones de las demás culturas existentes en su sociedad, y que desarrollen una actitud de respeto hacia ellas. Ver, Multiculturalism, Manuscrito (Madrid, 1992). En un sentido similar, ver, Amy Gutman, «The Challenge of Multiculturalism in Political Ethics,» Philosophy and Public Affairs, v.22 n.3 (1993). 
sobre la vida de los menores. Quiero decir, me interesa especialmente estudiar cómo responden las instituciones frente al hecho de que un menor, por el solo azar de haber nacido en una comunidad pobre, puede ver radicalmente afectada su vida. Así, por ejemplo, cómo reaccionan las instituciones respecto de la situación de un menor que tiene problemas (digamos) para recibir una adecuada alimentación; atención sanitaria; o formación intelectual, a raíz de hechos de los cuales el menor no es responsable en absoluto: simplemente ha nacido en un contexto pobre, en vez de en otro más rico.

$\mathrm{Al}$ respecto, cabe hacer una primera afirmación importante, en tren de proseguir con un análisis preferencial de cómo el Poder Judicial ha reaccionado en estos casos: por distintas razones, tales como la desconfianza en la justicia; la falta de incentivos adecuados; la carencia de recursos económicos; o la mera ignorancia; los pobres han encontrado enormes dificultades para ver sus problemas atendidos desde la esfera judicial. Esto es, sólo excepcionalmente la Justicia y los juristas norteamericanos se han abocado a estudiar temas vinculados a la pobreza ${ }^{28}$. Más difícil aún, entonces, resulta encontrar casos en los que se ponga de relieve el impacto de tales diferencias económicas sobre los menores. De todos modos, y a pesar de lo dicho, la Corte norteamericana no perdió la oportunidad de fijar algunos de sus criterios al respecto. En particular, ello ocurrió en un caso que se constituyó en uno de los más trascendentes de los resueltos por la Suprema Corte en los últimos tiempos, el caso «San Antonio Independent School District v. Rodríguez» (411 U.S. 1, de 1973). En esta ocasión, era discutida la constitucionalidad de una ley que provocaba fuertes desigualdades entre escuelas ubicadas en diferentes distritos. Esto, debido a que tales escuelas, conforme al estatuto en cuestión, se financiaban con impuestos que tomaban en cuenta los valores de la propiedad en tales diversos distritos. A raíz de ello, las escuelas ubicadas en las áreas más pobres, recibían mucho menores recursos que aquellas ubicadas en las áreas más privilegiadas. Conforme a la sentencia de la Corte, un estatuto como el que regía en San Antonio, Texas, no podía ser tildado de inconstitucional. Según la opinión de la mayoría, liderada por el juez Powell, los demandantes hubiesen tenido derecho a levantar tales reclamos, si el sistema de financiamiento implicase, para algunos menores, la absoluta imposibilidad de acceder a la escuela. En cambio -afirmaron- «cuando sólo están en juego diferencias relativas en materia de gastos», no existe ninguna base como para levantar una queja frente al estado.

Esta decisión que implicó desconocer a la educación su carácter de «derecho fundamental», produjo obvias reacciones en aquellos pensadores más cercanos a ideales igualitarios. Una de las más notables, de entre estas reacciones, apareció incluida en el mismo fallo, como opinión disidente, y fue

28 Entre estas notables excepciones, especialmente, resultan importantes los trabajos de Frank Michelman, tales como «On Protecting the Poor through the Foucteenth Amendment.» 83 Harvard Law Review 7 (1969); o «Welfare Rights in a Constitutional Democracy.»1979 Washington U.L.O. 659745. 
la del juez T. Marshall. Precisando algunas cuestiones que todo liberal igualitario hubiera procurado precisar, Marshall sostuvo la especial relevancia de la educación, dentro de una sociedad basada en la deliberación colectiva. Según él (y debido a la «directa e inmediata» conexión con la libertad de opinión, y el buen funcionamiento del proceso político), la educación y los modos en que ella era impartida, debían ser objeto de un particular escrutinio. Y, de acuerdo con estas pautas, Marshall entendía que en el caso se habían «seleccionado medios totalmente inapropiados para asegurar los fines» declarados en la ley. Según me parece, en su opinión, Marshall no hizo sino mostrar coherencia con sus creencias igualitarias, sugiriendo el camino que todo liberal igualitario podría sugerir. Este camino es, me parece, el de intentar remediar aquellos casos en los cuales alguien pueda verse afectado, a partir de situaciones que escapan a su responsabilidad.

\section{Posibles alcances del igualitarismo económico}

Ahora bien, me parece importante tratar de precisar con algún detalle mayor qué es lo que un liberal igualitario podría hacer, en casos como el citado en la sección anterior, para ayudar a los individuos más desfavorecidos de la sociedad. Una respuesta sencilla de enunciar pero, entiendo, algo apresurada, diría simplemente que lo que debe hacerse es mejorar especialmente la calidad de la educación que reciben quienes están peor. Sin embargo, creo que una visión realista de la sociedad tiene que movernos a ver cierta implausibilidad en esta solución. Según entiendo, el principal problema que enfrentan los/las menores miembros de comunidades pobres, respecto de la educación, es otro: los enormes incentivos que reciben para abandonar los estudios, más allá de la calidad de los mismos. Para decirlo mejor, por un lado me parece cierto que una mala educación puede resultar una razón importante (para los menores) que los mueve a abandonar estudios que aparecen como especialmente inútiles. De todos modos si los/las menores pobres dejan de estudiar, entiendo yo, eso se debe adicionalmente (y tal vez de un modo más relevante), a que sus mayores los presionan para que lo hagan, y así puedan contribuir al sostén económico de su familia o núcleo social. La pobre educación; la presión de padres o tutores; y la enorme tentación de largarse a la calle a recaudar algunas monedas, en conjunto, suman una serie importante de incentivos que mueven a los/las menores pobres a abandonar sus estudios.

Alguien podría criticar al enfoque anterior, sin embargo, señalando que la sociedad hace bastante para que la situación descrita no se presente, al contraofertarle a los menores «importantes compensaciones», si es que se deciden a continuar estudiando. Esto es, las sociedades modernas suelen premiar mucho mejor a aquellos de entre sus miembros que han estudiado; mientras que compensan bastante pobremente (en general) a aquellos que, digamos, se dedican a meras actividades manuales. Frente a esta replica, yo 
señalaría, entonces, lo siguiente: «el valle de la transición ${ }^{29}$ hasta la terminación de los estudios es tan costoso para los/las menores pobres, que el deseo de completar los estudios no puede sino transformarse en un deseo utópico. Quiero decir, para tales menores, la decisión de seguir estudiando implica costos enormes debido (entre otras razones) a los recursos que sus familias deben invertir en esos años; los recursos que ellos/ellas (y sus familias) dejan de percibir por no poder dedicarse de lleno al trabajo; etc.; a lo que se suman las incertidumbres que toda apuesta a largo plazo conlleva.

Frente a este tipo de encerrona en la que quedan sujetos los/las menores pobres, ¿qué tipo de soluciones específicas podría aconsejar un liberal igualitario? Responder más o menos responsablemente a esta pregunta, requeriría desarrollar cuestiones que exceden largamente los límites de este trabajo. Sin embargo, me interesaría al menos dejar planteadas algunas intuiciones fuertes que se me aparecen, a este respecto. Creo que, en principio, habría dos vías posibles a transitar, con el fin de atender a la situación de los menores pobres, incentivados como dije a abandonar la educación. La primera solución que se me ocurre sería la de reducir los costos del recorrer aquel «valle de la transición». Esto podría significar, por ejemplo, no sólo mejorar la calidad de la educación que reciben los pobres, sino además otorgar incentivos económicos «compensatorios» a quienes opten por seguir con sus estudios. Un sistema significativo de becas, u otro tipo de apoyos suplementarios, podrían ser útiles, en este sentido. Me arriesgaría a decir, de cualquier manera, que tomarse en serio esta alternativa implicaría estar dispuestos a transferir una cantidad extraordinaria de recursos económicos para tal propósito, lo cual torna esta solución, seguramente, muy complicada.

La segunda respuesta es aún más complicada que la primera pero, me parece, resulta más fiel a los ideales del liberalismo igualitario. Esta segunda respuesta lleva a cuestionar aquello que estaba implícito en la mencionada idea de «transición». Lo que se cuestiona aquí es el sentido mismo de premiar de modo tan disímil a quienes eligen un cierto camino frente a otras alternativas. $\mathrm{O}$, para acercarme más a los casos aquí tratados, se objeta el hecho de que las actividades que tienen «más a su alcance» los miembros de las comunidades pobres, estén tan ruinosamente recompensadas. Esto es, se objeta que los/las menores, por hechos de los que no son en absoluto responsables (por ejemplo, el tener que haber dejado los estudios; o el haberse formado en un ámbito educativo peor), puedan verse perjudicados en sus elecciones futuras (por ejemplo, a través de las compensaciones ruinosas que habitualmente se destinan para el pago de actividades menos técnicas, y normalmente más costosas en términos de esfuerzo físico). Para ejemplificar la idea aquí sugerida, permítaseme volver sobre el caso «San Antonio», analizado más arriba. En aquella situación, y a partir del modo desigualitario en que se recaudaban e invertían los impuestos, la comunidad de

${ }^{29}$ Esta expresión esta tomada de Adam Przeworski, Democracy and the Market. Cambridge U.P., 1991. 
Texas invertía 248 por alumna en Edgewood, mientras utilizaba 558 por alumna en Álamo Heights. Luego, las primeras recibían una educación mucho más deficitaria que las segundas, lo cual, sumado a la ruinosa situación de dicha comunidad, llevaba a muchas de las estudiantes de Edgewood a cambiar sus estudios por trabajos ocasionales. El hecho de que, a los pocos años, las alumnas provenientes de Álamo Heights reciban mucho mejores compensaciones que las de Edgewood, es el hecho que aquí impugno como moralmente inaceptable. Esto, simplemente, si se tiene en cuenta que se está castigando a las ex-alumnas de Edgewood por hechos de los que ellas son básicamente víctimas.

Una solución igualitaria para estos casos (y esto responde sólo a una serie de intuiciones que debiera desarrollar mucho más detenidamente), podría consistir en lo siguiente: i) Compensar de modo relativamente similar a las actividades que requieren mayor formación técnica y a las que requieren menor formación (abandonando así los abismos compensatorios que hoy suelen separar unos casos de otros); ii) sin perjuicio de que colectivamente se puedan decidir especiales incentivos para algunas actividades que se consideren especialmente relevantes (procurando así, darle mayor racionalidad a un tipo de decisiones que hoy parecen estar guiadas por principios más bien caprichosos). A título muy provisional, estos lineamientos me resultarían intuitivamente aceptables a fin de impedir injusticias con aquellos que, por razones que les son básicamente ajenas, recibieron una formación peor. Más aún, creo que principios como los citados podrían resultar valiosos aún en el hipotético caso de tener resuelto el problema de los menores peor formados. Quiero decir, tales principios podrían ser adecuados para cualquier sociedad igualitaria a la que, en términos generales, le importa que sus miembros puedan autorrealizarse eligiendo y desarrollando libremente su plan de vida. Para ejemplificar lo que digo, considérense casos como los siguientes: Si una persona debe abdicar de su preferencia por ser maestro, debido a la pobre compensación que los maestros habitualmente reciben; si una persona, a disgusto, opta por ser empresaria, porque allí los éxitos se compensan con cifras siderales; si una persona resigna hasta su dignidad para ser modelo o prostituta, dado que de ese modo puede conseguir recompensas fabulosas, en comparación con las que le dejarían otras actividades de su agrado; entonces, podríamos pensar que la sociedad que autoriza estas situaciones, atenta contra la autonomía de sus individuos miembros. Soluciones como la sugerida, entiendo yo, permitirían que los individuos tengan mayor libertad para elegir el modelo de vida que quieran, al reemplazar básicamente el (irracional) sistema de incentivos y desincentivos que caracteriza a las sociedades modernas. 